\title{
Dual-dispersion-regime dual-comb mode-locked laser
}

\author{
Maciej Kowalczyk,,${ }^{1,}$ tukasz A. SterczeWski, ${ }^{1}$ XUZhao Zhang,,${ }^{2,3}$ Valentin \\ PETROV, ${ }^{4}$ AND JAROSŁAW SOTOR ${ }^{1}$ \\ ${ }^{1}$ Laser \& Fiber Electronics Group, Faculty of Electronics, Photonics and Microsystems, Wroclaw University of Science and Technology, Wybrzeże \\ Wyspiańskiego 27, 50-370 Wrocław, Poland \\ ${ }^{2}$ State Key Laboratory of Crystal Materials, Shandong University, 250100 Jinan, China \\ ${ }^{3}$ College of Information Science and Technology, Qingdao University of Science and Technology, Qingdao 266061, China \\ ${ }^{4}$ Max Born Institute for Nonlinear Optics and Ultrafast Spectroscopy, Max-Born-Str. 2a, 12489 Berlin, Germany \\ *Corresponding author: m.kowalczyk@pwr.edu.pl
}

Manuscript dated 01/25/2022.

\begin{abstract}
We report on the first solid-state dual-comb mode-locked laser simultaneously operating in different dispersion regimes. Due to the intrinsic polarization-multiplexing in a birefringent $\mathrm{Yb}: \mathrm{Ca}_{3} \mathrm{NbGa}_{3} \mathrm{Si}_{2} \mathrm{O}_{14}$ (Yb:CNGS) gain medium, the laser emits two cross-polarized pulse trains with a repetition rate offset of $\sim 4.8 \mathrm{kHz}$ from a single cavity. We obtain dual pulse generation with 20 -fold difference in the duration, by setting the net cavity group delay dispersion to cross zero across the emission band of the employed gain medium. While the duration of the soliton-like pulses experiencing anomalous dispersion amounts to $117 \mathrm{fs}$, the second laser output that is spectrally located in the normal dispersion region, is strongly chirped with a pulse duration of $2360 \mathrm{fs}$.
\end{abstract}

Locking two independent oscillators requires significant effort [1] compared to the intrinsic emission of mutually coherent beams from a shared laser cavity. Consequently, single-cavity dual-comb lasers (SCDCLs) [2] have emerged as interesting alternatives to pairs of independent optical frequency combs synchronized via electronic feedback loops [3]. Progress in the SCDCL field is primarily fueled by applications in dual-comb spectroscopy (DCS) [4], which enables broadband and high-resolution spectral measurements at high rates across different spectral regions. For most free-running SCDCL, the central DCS requirements of dissimilar repetition rates and high mutual comb coherence are naturally satisfied [5]. Therefore, demonstrations of demanding picometer-resolution measurements of highfinesse microcavities [6] or molecular transitions at low pressure [7] are routinely possible with these sources.

The first single-cavity dual-color femtosecond solid-state (Ti:sapphire) laser was demonstrated as early as 1993 [8]. At that time, however, asynchronous (due to different group delay dispersion; GDD) dual combs were not of particular interest because researchers focused on synchronizing the wavelength-multiplexed pulse trains. Therefore, their potential for use in DCS, or more generally, asynchronous optical sampling remained long unnoticed. This changed in recent years when numerous schemes for common-cavity dual-comb generation have been demonstrated. In particular, fiber-based bidirectional [9-13] and polarizationmultiplexing $[7,14,15]$ pulse shaping has greatly extended the original dual-wavelength concept [16,17]. Although the most popular platform for realization of the SCDCL concept is still the optical fiber, solid-state (bulk) dual-comb lasers have also experienced remarkable growth [18-24]. Not only do they offer reduced nonlinearities permitting generation of highpower radiation [21,23], but also support ultrashort pulse duration [18] and exceptionally low-noise operation [24].

Whereas most studies have focused on the dual-comb generation mechanism, repetition rate tuning, and mutual stability characterization, some research groups have reported on diverse temporal and spectral patterns emerging in SCDCLs. These include nontrivial phenomena such as the bound soliton state [25] or the occurrence of asynchronous pulse trains forming in different dispersion regimes, which can be observed primarily in dual-wavelength SCDCLs. For instance, Wang et al. have reported the coexistence of a 830fs-long stretched pulse with a 19.8-ps-long chirped (dissipative) soliton in a $1.95 \mu \mathrm{m} \mathrm{Tm}$-doped fiber laser incorporating high third order dispersion [25]. Analogouslyfeatured operation in a dispersion-managed Er-doped fiber laser was also presented [26]. Finally, a $1.05 \mu \mathrm{m}$ dual-color $\mathrm{Yb}$-doped fiber laser with mechanical dispersion tuning reported by Fellinger et al. enabled either generation of two solitons or operation in different dispersion regimes: normal for the blue-shifted and anomalous for the red-shifted pulses [17]. Notably, coexistence of temporally diverse pulses in the same dispersion regime has also been observed in fiber lasers generating two solitons with strongly differing spectral bandwidths [27,28].

Although these reports present the feasibility of realizing temporally and spectrally diverse dual-comb patterns in 
a shared laser cavity, all were implemented using optical fiber technology. This is because fiber lasers exhibit significantly higher intracavity nonlinearities and dispersion compared with their bulk counterparts, which greatly promotes exotic mode-locking regimes. In this letter, we address this niche and present the simultaneous generation of spectrally and temporally dissimilar pulses in a polarization-multiplexed solid-state Yb:CNGS dual-comb laser. Rather than ensuring anomalous dispersion for soliton mode-locking for the two combs, we promote the formation of cross-polarized pulses in both dispersion regimes (anomalous and normal), which enabled us to obtain a 20-fold difference in the pulse widths (117 fs vs. $2360 \mathrm{fs}$ ). Importantly for dual-comb applications, a slight improvement in the relative timing stability has also been observed when compared with the all-anomalousdispersion scheme [24].

The schematic of the developed dual-comb laser cavity is shown in Fig. 1. As a gain medium we employ an antireflective (AR)-coated $\mathrm{Yb}(3 \%)$ :CNGS crystal. This langasite-type calcium niobium gallium silicate offers a remarkably broadband emission spectrum that supports generation of sub-50 fs pulses, as demonstrated in our previous works $[29,30]$. The crystal used here has dimensions of $3 \times 3 \times 4 \mathrm{~mm}^{3}$ and is cut with an angle $\theta=36^{\circ}$ between the normal propagation direction and the optical axis $c$. Both $c$ and one of the crystallographic $a$ axes of this trigonal crystal lie in the horizontal plane. The active element is pumped by a $1 \mathrm{~W}$, single mode fiber-coupled laser diode operating at $979.4 \mathrm{~nm}$. Due to the modest power delivered by the pump laser, no cooling of the active element is required. The pump waist within the crystal has a radius of $25 \mu \mathrm{m}$ (measured) and matches well the estimated cavity laser mode radius of $22 \mu \mathrm{m}$ (obtained using the ABCD matrix formalism). Stable modelocking is initiated by a semiconductor saturable absorber mirror (SESAM) with a $500 \mathrm{fs}$ recovery time and a $2.6 \%$ modulation depth. The output coupler has a transmission of $1 \%$. The laser cavity is approximately $190 \mathrm{~cm}$ long which dictates the $\sim 78.5 \mathrm{MHz}$ pulse repetition rate.

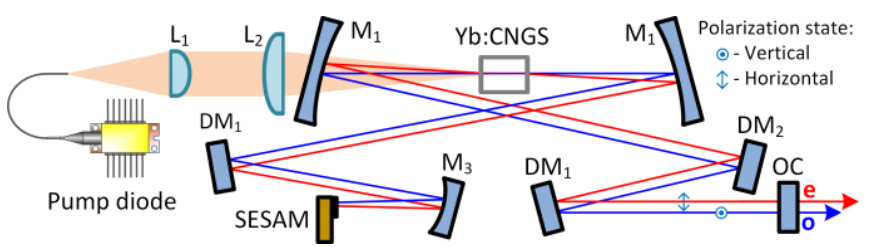

Fig. 1. Schematic of the dual-comb mode-locked Yb:CNGS laser. $\mathrm{L}_{1}-$ $18.4 \mathrm{~mm}$ aspheric lens; $\mathrm{L}_{2}-100 \mathrm{~mm}$ spherical lens; $\mathrm{M}_{1}-100 \mathrm{~mm}$ concave spherical mirrors; $\mathrm{DM}_{1,2}$ - dispersive mirrors; $\mathrm{OC}$ — output coupler; SESAM - semiconductor saturable absorber mirror; o/e beam - laser beams forming an ordinary/extraordinary ray inside the birefringent $\mathrm{Yb}$ :CNGS crystal.

One of the crucial aspects of the dual-dispersion laser design is clearly the estimation of the net cavity GDD. The major normal GDD contribution comes from the Yb:CNGS crystal introducing $+660 \mathrm{fs}^{2}$ and $+760 \mathrm{fs}^{2}$ per resonator roundtrip at a $1050 \mathrm{~nm}$ wavelength, respectively for the beam polarized as an ordinary $(o)$ - and an extraordinary $\left(e ; \theta=36^{\circ}\right)$ - wave [31]. This is further increased by two curved folding mirrors $M_{1}$ with a total round-trip GDD equal to $+400 \mathrm{fs}^{2}$ (simulation data provided by the manufacturer). Normal dispersion is counterbalanced by two types of dispersive mirrors $\left(\mathrm{DM}_{1,2}\right)$ with a total double-bounce GDD of $-1200 \mathrm{fs}^{2}$ (measurement data). Figure 2a shows the round-trip GDD curves for these components along with that of the SESAM. Unfortunately, the measurement data of the latter were provided by the manufacturer only up to $1050 \mathrm{~nm}$. No GDD data were available for $\mathrm{M}_{3}$ and $\mathrm{OC}$ mirrors except that their dispersive contributions should be negligible.

The developed laser supports dual-comb generation via intrinsic polarization-multiplexing [24]. Due to the employed special cut of the birefringent $\mathrm{Yb}$ :CNGS gain medium, one can align the cavity to achieve simultaneous stable lasing of both $o$ - and $e$-polarized beams. For that, the polarization of the pump radiation is set to form an $o$-ray inside the gain medium. Therefore, at normal incidence the pump and the $o$-polarized laser beam propagate collinearly inside the crystal. Once lasing of the $o$-beam is initiated, subsequent fine-alignment of the cavity end-mirrors allows one to trigger concurrent $e$-beam operation. Importantly, varying the mirrors' tilt provides full control over the power balance between the two generated beams, which is crucial regarding the $\mathrm{Yb}: \mathrm{CNGS}$ gain anisotropy for $\sigma$ (o-ray) and $\pi$ (e-ray) polarizations [32]. After obtaining dual-polarization continuous wave operation, a stable dual-comb mode-locked regime can be initiated with a mechanical perturbation of the SESAM.

Contrary to our previous work on a dual-comb laser emitting two soliton pulse trains [24], here we report simultaneous generation of pulses in two distinct dispersion regimes. Figure $2 b$ presents the optical spectra together with the estimated round-trip net cavity GDDs experienced by the two beams. In this analysis, we excluded the SESAM contribution due to the limited spectral range of the available data. However, its dispersion profile (see Fig. 2a) signifies a further enhancement of the GDD contrast experienced by the two combs. The $o$-beam spectrum exhibits a peak at $1062 \mathrm{~nm}$ with a corresponding GDD of approx. $-500 \mathrm{fs}^{2}$ (Fig. $2 b)$. The spectrum resembles a soliton $\left(\mathrm{sech}^{2}-\right)$ shape with characteristic Kelly sidebands. In contrast, the spectrum of the $e$-beam exhibits a rectangular profile typical for normal dispersion regime [33]. Indeed, the peak wavelength of $1032 \mathrm{~nm}$ corresponds to a GDD of $+400 \mathrm{fs}^{2}$.

The intensity autocorrelation measurement (Fig. 2c) yielded a pulse duration of $117 \mathrm{fs}$ for the $o$-beam, which is relatively close to its Fourier limit (time-bandwidth product (TBP) of 0.44$)$. On the contrary, the duration of the $e$-beam pulses amounted to $2.36 \mathrm{ps}(\mathrm{TBP}=5.92)$, indicating the presence of strong chirp. This confirms that the two combs experienced completely different pulse formation mechanisms, despite being simultaneously emitted from the very same laser cavity. Nevertheless, they maintained dualcomb properties with a repetition rate offset $\left(\Delta f_{\text {rep }}\right)$ of $4795 \mathrm{~Hz}$, as shown in the radio-frequency (RF) spectrum (Fig. 2d). The additional low intensity peaks spaced by multiples of $\Delta f_{\text {rep }}$ arise due to parasitic intermodulation of the 
main beat notes in the photodetector with limited linearity. The essential laser output parameters are summarized in Table 1.

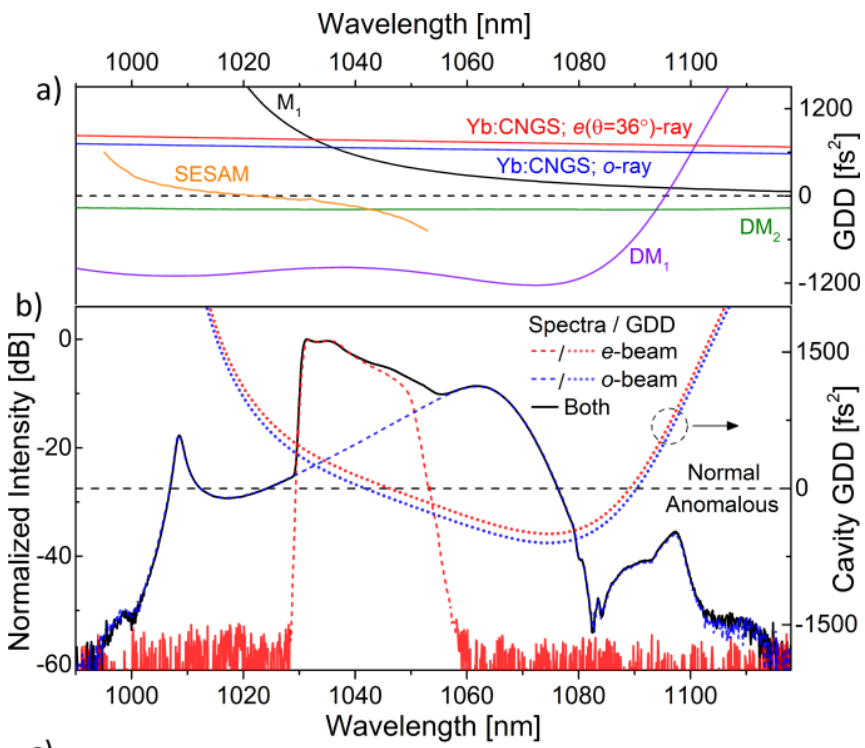

c)
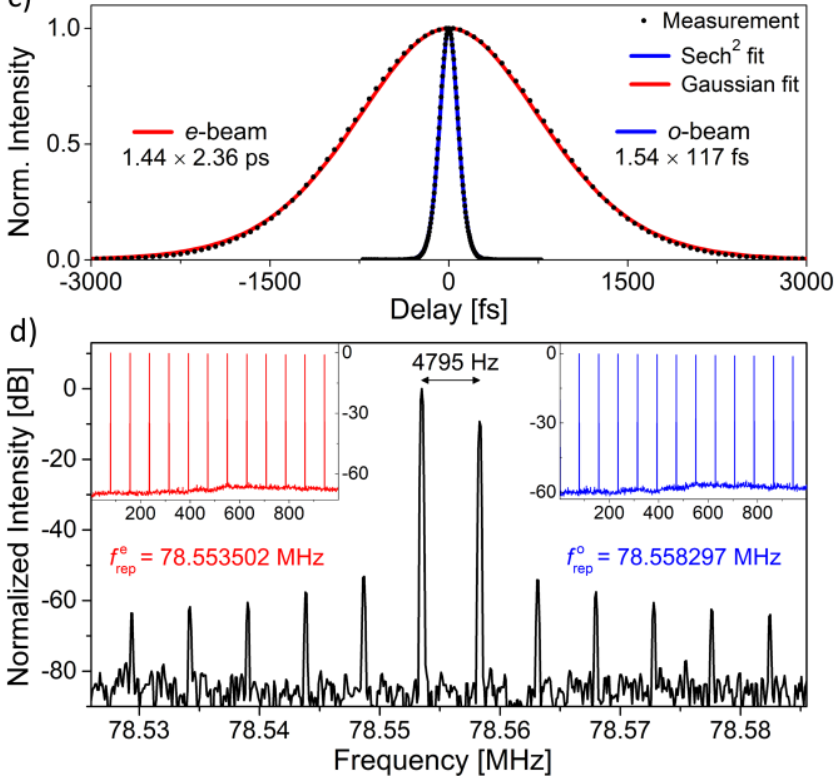

Fig. 2. (a) Round-trip GDD introduced by individual cavity components (two items of $\mathrm{M}_{1}$ and $\mathrm{DM}_{1}$ mirrors are implemented in the cavity and thus their contributions are doubled). Performance of the dual-comb mode-locked Yb:CNGS laser: (b) optical spectra of the $e / o$-beams and their superposition (logarithmic scale) together with the estimated round-trip net cavity GDD (excluding SESAM contribution); (c) corresponding autocorrelation traces with fits assuming sech ${ }^{2}$ - (o-beam) and Gaussian-shaped (e-beam) pulses; (d) corresponding RF spectra of the fundamental beat notes of both beams around $78.555 \mathrm{MHz}$ (resolution bandwidth; RBW=100 Hz); insets: 1-GHzwide $\mathrm{RF}$ spectra of the individual beams ( $\mathrm{RBW}=1 \mathrm{kHz})$.

To further confirm that the laser simultaneously emits two cross-polarized beams rather than operates in a polarizationswitching regime, we measured mutual beating between the generated combs in a simple dual-comb experiment. After separating the two beams by a polarizing beam-splitter, the polarization state of one of them is rotated with a half-wave plate for maximizing the interference signal. Subsequently, the beams are recombined with a 50:50 beam-splitter and pass through a home-built grating-based spectral bandpass filter. This enabled selection of narrowband radiation $(\Delta \lambda=0.8 \mathrm{~nm})$ to avoid aliasing of the down-converted comb. The two beams are then incident on a photodiode producing the beating signal, which is next amplified and low-pass filtered before acquiring with a fast oscilloscope in a similar way to that of ref. [24]. Figure 3 depicts an unprocessed trace of the registered electrical signal comprising a train of dual-comb interferograms with periodic bursts. Their temporal separation is defined by the inverse of the repetition rate offset $\Delta f_{\text {rep }}$ of $208 \mu$ s. The residual low-intensity spurious peaks visible between the consecutive bursts originate from parasitic intracavity reflections appearing due to imperfect AR-coatings of the gain crystal [24].

Table 1. Parameters of the output $e / o$-beams. $\lambda_{0}-$ peak emission wavelength; $\Delta \lambda$ - full-width-at-half-maximum (FWHM) spectral bandwidth; $\tau$ - FWHM pulse duration; TBP - time-bandwidth product; $P_{\mathrm{av}}$ - average output power; $f_{\text {rep }}$ - repetition rate.

\begin{tabular}{|c|c|c|}
\hline & $e$-beam & $o$-beam \\
\hline$\lambda_{0}$ & $1031.7 \mathrm{~nm}$ & $1061.7 \mathrm{~nm}$ \\
\hline$\Delta \lambda$ & $8.9 \mathrm{~nm}$ & $14 \mathrm{~nm}$ \\
\hline$\tau$ & $2360 \mathrm{fs}$ & $117 \mathrm{fs}$ \\
\hline $\mathrm{TBP}$ & 5.92 & 0.44 \\
\hline$P_{\mathrm{av}}$ & $80 \mathrm{~mW}$ & $20 \mathrm{~mW}$ \\
\hline$f_{\text {rep }}$ & $78.553502 \mathrm{MHz}$ & $78.558297 \mathrm{MHz}$ \\
\hline
\end{tabular}

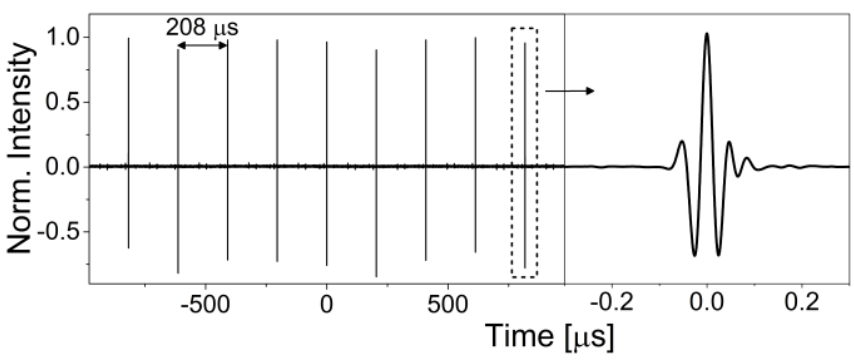

Fig. 3. A raw oscilloscope trace of the dual-comb interferogram with bursts spaced by $1 / \Delta f_{\text {rep }}=208 \mu \mathrm{s}$. One of the centerbursts is zoomed on the right.

For a more detailed comparison of the dual-dispersion dual-comb regime with the original all-anomalous-dispersion design [24] we studied their noise properties. Table 2 summarizes the most important parameters with the terminology adopted from ref. [34]: the relative RMS timing jitter between the two pulse trains $\left(\tau^{\mathrm{RMS}}\right)$, period timing jitter $\left(\tau^{\text {period }}\right)$, and integrated phase noise of the relative carrierenvelope-offset frequency $\left(\int \mathrm{S}_{\triangle f c e o}\right.$; see Supplemental Document for detailed explanation and additional data). We found out that the presented laser exhibits an improvement in repetition rate stability, while its relative $f_{\text {ceo }}$ noise remains virtually the same. We expect that this noise behavior may be attributed to the reduced spectral overlap between the combs around their central wavelengths (leading to a suppressed gain-competition-based cross-coupling) as well as 
a significantly lower peak power of the $e$-beam (which weakens nonlinear cross-coupling). Nevertheless, the exact explanation of this phenomenon requires further, more detailed investigations, which may be provided by pulse-topulse-resolved mode-locking dynamics studies [33,35], that will be covered by our future works.

Table 2. Comparison of relative stability performance between the all-anomalous [24] and dual-dispersion mode of operation of the Yb:CNGS dual-comb laser. $\tau^{\mathrm{RMS}}-$ relative RMS timing jitter between the two pulse trains; $\tau^{\text {period }}$ — period timing jitter, $\int \mathrm{S}_{\Delta \text { fceo }}$ -

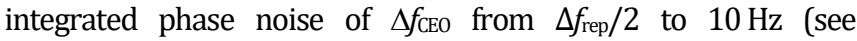
Supplemental Document for details).

\begin{tabular}{|c|c|c|}
\hline & $\begin{array}{c}\text { All-anomalous } \\
{[24]}\end{array}$ & $\begin{array}{c}\text { Dual-dispersion [this } \\
\text { work] }\end{array}$ \\
\hline$\tau^{\text {RMS }}$ & $20.7 \mathrm{ps}$ & $2.5 \mathrm{ps}$ \\
\hline$\tau^{\text {period }}$ & $331.3 \mathrm{fs}$ & $108.8 \mathrm{fs}$ \\
\hline $\int \mathrm{S}_{\Delta \text { fceo }}$ & $9.7 \mathrm{rad}$ & $11.2 \mathrm{rad}$ \\
\hline
\end{tabular}

In conclusion, we have demonstrated a mode-locked bulk solid-state laser simultaneously emitting two combs that exploits polarization-multiplexing in the Yb:CNGS gain medium. The net cavity GDD crossing zero across the laser emission band allowed generation of the individual combs in different dispersion regimes. Consequently, despite being simultaneously emitted from the very same laser cavity, the two orthogonally polarized pulse trains experienced distinct pulse formation mechanisms resulting in dissimilar temporal and spectral profiles. We achieved a 20 -fold pulse duration difference with $117 \mathrm{fs}$ and $2360 \mathrm{fs}$ for the pulses shaped in the presence of anomalous and normal dispersion, respectively. The presented laser source opens a novel avenue for studying fundamental mode-locking dynamics by providing insight into interplay between pulses sharing the very same bulk laser cavity and gain medium, yet experiencing completely different pulse formation mechanisms [33, 35-37]. Moreover, we expect that the observed improvement of the relative timing jitter with respect to the dual-soliton regime [24] may help to develop more stable free-running single-cavity dualcomb lasers in the future.

Funding. National Science Centre (NCN, Poland) (2015/18/E/ST7/00296); Horizon 2020 Framework Programme (H2020) (101027721).

Acknowledgments. We would like to acknowledge Laser Zentrum Hannover for fabrication of the AR coatings on the Yb:CNGS sample. This project has received funding from the European Union's Horizon 2020 research and innovation programme under the Marie Skłodowska-Curie grant agreement No 101027721.

Disclosures. The authors declare no conflicts of interest.

Data availability. Data underlying the results presented in this letter may be obtained from the authors upon reasonable request.

Supplemental document. See Supplement 1 for supporting content.

\section{References}

1. I. Coddington, W. C. Swann, and N. R. Newbury, Phys. Rev. Lett. 100, 013902 (2008).

2. R. Liao, H. Tian, W. Liu, R. Li, Y. Song, and M. Hu, J. Phys. Photonics 2, 042006 (2020).
3. N. Picqué and T. W. Hänsch, Nat. Photonics 13, 146 (2019).

4. I. Coddington, N. Newbury, and W. Swann, Optica 3, 414 (2016).

5. S. M. Link, D. Maas, D. Waldburger, and U. Keller, Science 356, 1164 (2017).

6. X. Zhao, G. Hu, B. Zhao, C. Li, Y. Pan, Y. Liu, T. Yasui, and Z. Zheng, Opt. Express 24, 21833 (2016).

7. Ł. A. Sterczewski, A. Przewłoka, W. Kaszub, and J. Sotor, APL Photonics 4, 116102 (2019).

8. M. R. X. de Barros and P. C. Becker, Opt. Lett. 18, 631 (1993).

9. K. Kieu and M. Mansuripur, Opt. Lett. 33, 64 (2008).

10. V. Mamidala, R. I. Woodward, Y. Yang, H. H. Liu, and K. K. Chow, Opt. Express 22, 4539 (2014).

11. S. Mehravar, R. A. Norwood, N. Peyghambarian, and K. Kieu, Appl. Phys. Lett. 108, 231104 (2016).

12. M. I. Kayes, N. Abdukerim, A. Rekik, and M. Rochette, Opt. Lett. 43, 5809 (2018).

13. B. Li, J. Xing, D. Kwon, Y. Xie, N. Prakash, J. Kim, and S.-W. Huang, Optica 7, 961 (2020).

14. A. E. Akosman and M. Y. Sander, Opt. Express 25, 18592 (2017).

15. X. Zhao, T. Li, Y. Liu, Q. Li, and Z. Zheng, Photonics Res. 6, 853 (2018).

16. J. Fellinger, G. Winkler, A. S. Mayer, L. R. Steidle, and O. H. Heckl, Opt. Express 27, 5478 (2019).

17. J. Fellinger, A. S. Mayer, G. Winkler, W. Grosinger, G.-W. Truong, S. Droste, C. Li, C. M. Heyl, I. Hartl, and O. H. Heckl, Opt. Express 27, 28062 (2019).

18. T. Ideguchi, T. Nakamura, Y. Kobayashi, and K. Goda, Optica 3, 748 (2016).

19. S. M. Link, A. Klenner, M. Mangold, C. A. Zaugg, M. Golling, B. W. Tilma, and U. Keller, Opt. Express 23, 5521 (2015).

20. M. T. Chang, H. C. Liang, K. W. Su, and Y. F. Chen, Opt. Express 23, 10111 (2015).

21. K. Fritsch, J. Brons, M. Iandulskii, K. F. Mak, Z. Chen, N. Picqué, and O. Pronin, arXiv preprint:2004.10303 (2020).

22. B. Willenberg, B. Willenberg, J. Pupeikis, J. Pupeikis, L. M. Krüger, F. Koch, C. R. Phillips, and U. Keller, Opt. Express 28, 30275 (2020).

23. N. Modsching, J. Drs, P. Brochard, J. Fischer, S. Schilt, V. J. Wittwer, and T. Südmeyer, Opt. Express 29, 15104 (2021).

24. M. Kowalczyk, Ł. Sterczewski, X. Zhang, V. Petrov, Z. Wang, and J. Sotor, Laser \& Photonics Rev. 15, 2000441 (2021)

25. Y. Wang, J. Li, L. Hong, G. Li, F. Liu, X. Zhou, and Y. Liu, Opt. Express 26, 18190 (2018).

26. X. Jin, M. Zhang, G. Hu, G. Hu, Q. Wu, Z. Zheng, Z. Zheng, and T. Hasan, Opt. Express 28, 6937 (2020).

27. Y. Liu, X. Zhao, G. Hu, C. Li, B. Zhao, and Z. Zheng, Opt. Express 24, 21392 (2016).

28. D. D. Han, X. M. Liu, Y. D. Cui, G. X. Wang, C. Zeng, and L. Yun, Opt. Lett. 39, 1565 (2014).

29. M. Kowalczyk, X. Zhang, X. Mateos, S. Guo, Z. Wang, X. Xu, P. Loiko, J. E. Bae, F. Rotermund, J. Sotor, U. Griebner, and V. Petrov, Opt. Express 27, 590 (2019).

30. M. Kowalczyk, X. Zhang, X. Zhang, V. Petrov, P. Loiko, X. Mateos, S. Guo, Z. Wang, X. Xu, and J. Sotor, J. Opt. Soc. Am. B 37, 2822 (2020).

31. X. Zhang, X. Zhang, S. Guo, J. He, K. Han, F. Lou, B. Zhang, R. Wang, and X. Liu, Opt. Mater. Express 5, 977 (2015).

32. X. Zhang, P. Loiko, J. M. Serres, X. Mateos, J. Ren, Z. Wang, S. Guo, X. Xu, E. Vilejshikova, U. Griebner, V. Petrov, M. Aguiló, and F. Díaz, Opt. Mater. Express 7, 3626 (2017).

33. P. Grelu and N. Akhmediev, Nat. Photonics 6, 84 (2012).

34. S. L. Camenzind, D. Koenen, B. Willenberg, J. Pupeikis, C. R. Phillips, and U. Keller, arXiv preprint: 2111.06085 (2021).

35. A. Mahjoubfar, D. V. Churkin, S. Barland, N. Broderick, S. K. Turitsyn, and B. Jalali, Nat. Photonics 11, 341 (2017).

36. Y. Wei, B. Li, X. Wei, Y. Yu, and K. K. Y. Wong, Appl. Phys. Lett. 112, 081104 (2018).

37. A. E. Akosman, J. Zeng, P. D. Samolis, and M. Y. Sander, IEEE J. Sel. Top. Quantum Electron. 24, 1 (2018). 


\section{Full references}

1. I. Coddington, W. C. Swann, and N. R. Newbury, "Coherent multiheterodyne spectroscopy using stabilized optical frequency combs," Phys. Rev. Let. 100, 013902 (2008).

2. R. Liao, H. Tian, W. Liu, R. Li, Y. Song, and M. Hu, "Dual-comb generation from a single laser source: principles and spectroscopic applications towards mid-IR-A review," J. Phys. Photonics 2, 042006 (2020).

3. N. Picqué and T. W. Hänsch, "Frequency comb spectroscopy," Nat. Photonics 13, 146-157 (2019).

4. I. Coddington, N. Newbury, and W. Swann, "Dual-comb spectroscopy," Optica 3, 414-426 (2016).

5. S. M. Link, D. Maas, D. Waldburger, and U. Keller, "Dual-comb spectroscopy of water vapor with a free-running semiconductor disk laser," Science 356, 1164-1168 (2017).

6. X. Zhao, G. Hu, B. Zhao, C. Li, Y. Pan, Y. Liu, T. Yasui, and Z. Zheng, "Picometer-resolution dual-comb spectroscopy with a free-running fiber laser," Opt. Express 24, 21833-21845 (2016).

7. Ł. A. Sterczewski, A. Przewłoka, W. Kaszub, and J. Sotor, "Computational Doppler-limited dual-comb spectroscopy with a free-running all-fiber laser," APL Photonics 4, 116102 (2019).

8. M. R. X. de Barros and P. C. Becker, "Two-color synchronously mode-locked femtosecond Ti:sapphire laser," Opt. Lett. 18, 631633 (1993).

9. K. Kieu and M. Mansuripur, "All-fiber bidirectional passively modelocked ring laser," Opt. Lett. 33, 64-66 (2008).

10. V. Mamidala, R. I. Woodward, Y. Yang, H. H. Liu, and K. K. Chow, "Graphene-based passively mode-locked bidirectional fiber ring laser," Opt. Express 22, 4539-4546 (2014).

11. S. Mehravar, R. A. Norwood, N. Peyghambarian, and K. Kieu, "Realtime dual-comb spectroscopy with a free-running bidirectionally mode-locked fiber laser," Appl. Phys. Lett. 108, 231104 (2016).

12. M. I. Kayes, N. Abdukerim, A. Rekik, and M. Rochette, "Freerunning mode-locked laser based dual-comb spectroscopy," Opt. Lett. 43, 5809 (2018).

13. B. Li, J. Xing, D. Kwon, Y. Xie, N. Prakash, J. Kim, and S.-W. Huang, "Bidirectional mode-locked all-normal dispersion fiber laser," Optica 7, 961-964 (2020).

14. A. E. Akosman and M. Y. Sander, "Dual comb generation from a mode-locked fiber laser with orthogonally polarized interlaced pulses," Opt. Express 25, 18592-18602 (2017).

15. X. Zhao, T. Li, Y. Liu, Q. Li, and Z. Zheng, "Polarization-multiplexed, dual-comb all-fiber mode-locked laser," Photonics Res. 6, 853-857 (2018).

16. J. Fellinger, G. Winkler, A. S. Mayer, L. R. Steidle, and O. H. Heckl, "Tunable dual-color operation of $\mathrm{Yb}$ : fiber laser via mechanical spectral subdivision," Opt. Express 27, 5478-5486 (2019).

17. J. Fellinger, A. S. Mayer, G. Winkler, W. Grosinger, G.-W. Truong, S. Droste, C. Li, C. M. Heyl, I. Hartl, and O. H. Heckl, "Tunable dualcomb from an all-polarization-maintaining single-cavity dual-color Yb: fiber laser," Opt. Express 27, 28062-28074 (2019).

18. T. Ideguchi, T. Nakamura, Y. Kobayashi, and K. Goda, "Kerr-lens mode-locked bidirectional dual-comb ring laser for broadband dual-comb spectroscopy," Optica 3, 748-753 (2016).

19. S. M. Link, A. Klenner, M. Mangold, C. A. Zaugg, M. Golling, B. W. Tilma, and U. Keller, "Dual-comb modelocked laser," Opt. Express 23, 5521-5531 (2015).

20. M. T. Chang, H. C. Liang, K. W. Su, and Y. F. Chen, "Dual-comb selfmode-locked monolithic $\mathrm{Yb}: \mathrm{KGW}$ laser with orthogonal polarizations," Opt. Express 23, 10111-10116 (2015).

21. K. Fritsch, J. Brons, M. landulskii, K. F. Mak, Z. Chen, N. Picqué, and O. Pronin, "Dual-comb thin-disk oscillator," arXiv preprint: 2004.10303 (2020).
22. B. Willenberg, B. Willenberg, J. Pupeikis, J. Pupeikis, L. M. Krüger, F. Koch, C. R. Phillips, and U. Keller, "Femtosecond dual-comb Yb:CaF laser from a single free-running polarization-multiplexed cavity for optical sampling applications," Opt. Express 28, 30275-30288 (2020).

23. N. Modsching, J. Drs, P. Brochard, J. Fischer, S. Schilt, V. J. Wittwer, and T. Südmeyer, "High-power dual-comb thin-disk laser oscillator for fast high-resolution spectroscopy," Opt. Express 29, 1510415113 (2021).

24. M. Kowalczyk, Ł. Sterczewski, X. Zhang, V. Petrov, Z. Wang, and J. Sotor, "Dual-Comb Femtosecond Solid-State Laser with Inherent Polarization-Multiplexing," Laser \& Photonics Rev. 15, 2000441 (2021).

25. Y. Wang, J. Li, L. Hong, G. Li, F. Liu, X. Zhou, and Y. Liu, "Coexistence of dissipative soliton and stretched pulse in dual-wavelength modelocked Tm-doped fiber laser with strong third-order dispersion," Opt. Express 26, 18190-18201 (2018).

26. X. Jin, M. Zhang, G. Hu, G. Hu, Q. Wu, Z. Zheng, Z. Zheng, and T. Hasan, "Broad bandwidth dual-wavelength fiber laser simultaneously delivering stretched pulse and dissipative soliton," Opt. Express 28, 6937-6944 (2020).

27. Y. Liu, X. Zhao, G. Hu, C. Li, B. Zhao, and Z. Zheng, "Unidirectional, dual-comb lasing under multiple pulse formation mechanisms in a passively mode-locked fiber ring laser," Opt. Express 24, 21392 (2016).

28. D. D. Han, X. M. Liu, Y. D. Cui, G. X. Wang, C. Zeng, and L. Yun, "Simultaneous picosecond and femtosecond solitons delivered from a nanotube-mode-locked all-fiber laser," Opt. Lett. 39, 15651568 (2014).

29. M. Kowalczyk, X. Zhang, X. Mateos, S. Guo, Z. Wang, X. Xu, P. Loiko, J. E. Bae, F. Rotermund, J. Sotor, U. Griebner, and V. Petrov, "Graphene and SESAM mode-locked Yb:CNGS lasers with selffrequency doubling properties," Opt. Express 27, 590-596 (2019).

30. M. Kowalczyk, X. Zhang, X. Zhang, V. Petrov, P. Loiko, X. Mateos, S. Guo, Z. Wang, X. Xu, and J. Sotor, "Self-frequency-doubling $\mathrm{Yb}: \mathrm{CNGS}$ lasers operating in the femtosecond regime," J. Opt. Soc. Am. B 37, 2822-2829 (2020).

31. X. Zhang, X. Zhang, S. Guo, J. He, K. Han, F. Lou, B. Zhang, R. Wang, and X. Liu, "Growth and optical properties of a new CGG-type laser crystal Nd ${ }^{3+}$ :CNGS," Opt. Mater. Express 5, 977-985 (2015).

32. X. Zhang, P. Loiko, J. M. Serres, X. Mateos, J. Ren, Z. Wang, S. Guo, X. Xu, E. Vilejshikova, U. Griebner, V. Petrov, M. Aguiló, and F. Díaz, "Highly-efficient laser operation of a novel trigonal silicate crystal $\mathrm{Yb}^{3+}: \mathrm{Ca}_{3} \mathrm{NbGa}_{3} \mathrm{Si}_{2} \mathrm{O}_{14}$," Opt. Mater. Express 7, 3626-3633 (2017).

33. P. Grelu and N. Akhmediev, "Dissipative solitons for mode-locked lasers," Nat. Photonics 6, 84-92 (2012).

34. S. L. Camenzind, D. Koenen, B. Willenberg, J. Pupeikis, C. R. Phillips, and $U$. Keller, "Timing jitter characterization of free-running dualcomb laser with sub-attosecond resolution using optical heterodyne detection", arXiv preprint: 2111.06085 (2021).

35. A. Mahjoubfar, D. V. Churkin, S. Barland, N. Broderick, S. K. Turitsyn, and B. Jalali, "Time stretch and its applications," Nat. Photonics 11, 341-351 (2017).

36. Y. Wei, B. Li, X. Wei, Y. Yu, and K. K. Y. Wong, "Ultrafast spectral dynamics of dual-color-soliton intracavity collision in a modelocked fiber laser," Appl. Phys. Lett. 112, 081104 (2018).

37. A. E. Akosman, J. Zeng, P. D. Samolis, and M. Y. Sander, "Polarization Rotation Dynamics in Harmonically Mode-Locked Vector Soliton Fiber Lasers," IEEE J. Sel. Top. Quantum Electron. 24, 1-7 (2018). 


\section{Dual-dispersion-regime dual-comb mode- locked laser: supplemental document}

\section{NOISE CHARACTERISTICS}

The different pulse shaping mechanisms of the dual-dispersion dual-comb laser presented in the primary document may influence the relative noise characteristics compared to the original all-anomalous-dispersion dual-comb laser design of ref. [1]. In particular, it is unclear how the two setups compare in terms of common phase noise suppression relevant for mode-resolved dual-comb spectroscopy. Because the relative timing and carrier-envelope offset (CEO) noise can be inferred from dual-comb interferograms, we processed 200-ms-long acquisitions using a computational phase retrieval and correction algorithm [2] to compare the two setups.

First we compare the timing stability, where the nomenclature of ref. [3] by Camenzind et al. is adopted. The relative root-mean-square (RMS) timing jitter $\tau^{\mathrm{RMS}}$ is calculated from the $n$-th interferogram arriving at $t_{n}$, and compared to the expected time-of-arrival (TOA) defined by multiples of the expected period $1 /\left\langle\Delta f_{\text {rep }}\right\rangle$ :

$$
\tau^{\mathrm{RMS}}[n]=t_{n}-\frac{n}{\left\langle\Delta f_{\text {rep }}\right\rangle} .
$$

This quantity characterizes how much the delay between optical pulses from the two combs fluctuates compared to the jitter-free case. To retrieve the TOAs needed for the characterization, we employ a constant fraction discriminator [2], which provides a numerical trigger based on the digital difference frequency signal (DDFG) [4]. However, other techniques should serve this application equally well like detecting peaks of the dual-comb signal envelope [3] or finding the maximum of the cross-correlation/cross-ambiguity function [5,6]. Note that to relate the microwave and optical domains, we employ a comb factor $\Delta f_{\text {rep }} / f_{\text {rep }}$ in our calculations, as in ref. [3].

Another related quantity under study is the period timing jitter, which measures how much the duration of interferograms (difference of consecutive TOAs) fluctuates relative to the expected period. It is defined as

$$
\tau^{\text {period }}[n]=\left(t_{n}-t_{n-1}\right)-\frac{1}{\left\langle\Delta f_{\text {rep }}\right\rangle} .
$$

Table S1 summarizes the numerical results obtained for the two lasers.

Table S1. Comparison between the all-anomalous and dual-dispersion mode of operation of the dualcomb mode-locked laser utilizing a Yb:CNGS gain medium.

\begin{tabular}{|c|c|c|}
\hline & $\begin{array}{c}\text { Single-dispersion } \\
\text { (all-anomalous) regime [1] }\end{array}$ & Dual-dispersion-regime \\
\hline $\begin{array}{c}\text { Relative RMS timing jitter } \\
\text { between the two pulse trains }\end{array}$ & $20.7 \mathrm{ps}$ & $2.5 \mathrm{ps}$ \\
\hline Period timing jitter & $331.3 \mathrm{fs}$ & $108.8 \mathrm{fs}$ \\
\hline
\end{tabular}

Unexpectedly, we find that both the relative RMS timing jitter, and period timing jitter greatly improve when we employ the dual-dispersion setup. Consequently, the latter is better suited for free-running asynchronous optical sampling or dual-comb spectroscopy experiments. The exact origin of the noise performance improvement needs further research but we postulate 
that it may relate to (i) reduced spectral overlap between the combs; (ii) lower peak power of the multi-ps pulse that forms in the normal dispersion regime. When the two pulses temporally overlap inside the gain crystal, they compete for the gain. This often leads to severe pulse amplitude modulation, which manifests itself in polarization-multiplexed fiber lasers as slowlyvarying post-centerburst oscillations in the dual-comb interferogram [7]. Here, the spectral overlap between the $o$ - and $e$-beams around their respective peak wavelengths is significantly smaller when compared to ref. [1], which might have contributed to decreasing their gaincompetition-based cross-coupling. Additionally, by lengthening the duration of one of the pulses to the multi-picosecond range, we expect to have greatly suppressed intensity-dependent inter-pulse nonlinear interaction.. Note that it is minor sub-Hz $\Delta f_{\text {rep }}$ drifts that are mostly responsible for the higher jitter reported here, which correspond to a relative $f_{\text {rep }}$ stability as low as $10^{-8}$. We also want to underline that the numbers quoted for the two free-running dual-comb laser setups are not a limitation per se, and can be greatly improved with a more robust mechanical cavity design [3].

Equally important for performance evaluation is the phase of the relative carrier-envelopeoffset (CEO) frequency ( $\Delta f_{\text {CEO }}$ ), or simply the interferogram carrier-envelope phase $\Delta \varphi_{0}$. For a stable dual-comb laser with $\Delta f_{\mathrm{CEO}} \neq 0, \Delta \varphi_{0}$ is expected to evolve linearly. Unfortunately, a freerunning non-CEO-stabilized dual-comb laser exhibits highly nonlinear phase excursions instead. In Fig. S1, we characterize the power spectral density (PSD) of computationallyretrieved $\Delta \varphi_{0}$ compensated for the linear trend. The top panel illustrates the frequency spectrum (power spectral density) of phase excursions, while the bottom shows the frequency-integrated phase noise retrieved from the PSD $\left(\int \mathrm{S}_{\Delta f c e o}\right)$. Note that $\Delta \varphi_{0}$ is sampled only around interferogram centerbursts with a sampling frequency of $\Delta f_{\text {rep }}$, which limits the analysis frequency range to $\Delta f_{\text {rep }} / 2$.

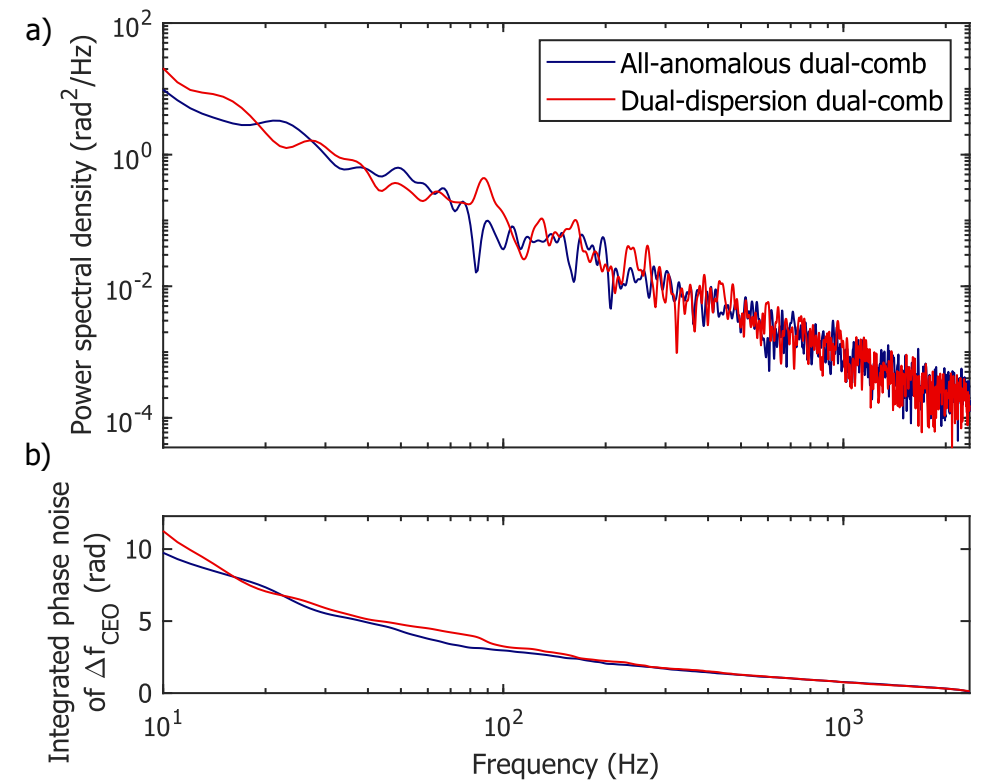

Fig. S1. Relative offset frequency noise characterization retrieved from dual-comb interferograms for the anomalous-only dispersion regime laser [1], and the dualdispersion regime laser discussed in the main manuscript. (a) Power spectral density (PSD) of relative CEO phase fluctuations. (b) Integrated phase noise retrieved from the PSD.

Unlike in the timing jitter case, we do not see significant differences between the two laser configurations. Both display nearly the same relative carrier-envelope phase noise 
characteristics with a dominant $1 / f^{2}$ slope (white frequency noise rolling off $-20 \mathrm{~dB} / \mathrm{decade}$ ) and only minor local differences occurring at acoustic/mechanical noise frequencies. This further confirms the importance of stable mechanical cavity design for low-timing-jitter and low-phase-noise operation, which may be potentially degraded by environmental noise effects.

\section{References}

1. M. Kowalczyk, Ł. Sterczewski, X. Zhang, V. Petrov, Z. Wang, and J. Sotor, "DualComb Femtosecond Solid-State Laser with Inherent Polarization-Multiplexing," Laser Photonics Rev. 15, 2000441 (2021).

2. $\quad$ Ł. A. Sterczewski, A. Przewłoka, W. Kaszub, and J. Sotor, "Computational Dopplerlimited dual-comb spectroscopy with a free-running all-fiber laser," APL Photonics 4, 116102 (2019).

3. S. L. Camenzind, D. Koenen, B. Willenberg, J. Pupeikis, C. R. Phillips, and U. Keller, "Timing jitter characterization of free-running dual-comb laser with sub-attosecond resolution using optical heterodyne detection," arXiv preprint: 2111.06085 (2021).

4. L. A. Sterczewski, J. Westberg, and G. Wysocki, "Computational coherent averaging for free-running dual-comb spectroscopy," Opt. Express 27, 23875-23893 (2019).

5. N. B. Hébert, J. Genest, J.-D. Deschênes, H. Bergeron, G. Y. Chen, C. Khurmi, and D. G. Lancaster, "Self-corrected chip-based dual-comb spectrometer," Opt. Express 25, 8168-8179 (2017).

6. N. B. Hébert, V. Michaud-Belleau, J.-D. Deschênes, and J. Genest, "Self-Correction Limits in Dual-Comb Interferometry," IEEE J. Quantum Electron. 55, 1-11 (2019).

7. Z. Deng, Y. Liu, C. Ouyang, W. Zhang, C. Wang, and W. Li, "Mutually coherent dualcomb source generated from a free-running linear fiber laser," Results Phys. 14, 102364 (2019). 